\title{
miR-454-3p suppresses cell migration and invasion by targeting CPEB1 in human glioblastoma
}

\author{
$\mathrm{XIAOBO} \mathrm{HUI}^{1,2}$, SHIMING $\mathrm{ZHANG}^{2}$ and YANPING WANG ${ }^{1}$ \\ ${ }^{1}$ Department of Neurosurgery, Huai'an First People's Hospital, Nanjing Medical University, Huai'an, Jiangsu 223300; \\ ${ }^{2}$ Department of Neurosurgery, The First Affiliated Hospital of Soochow University, Suzhou, Jiangsu 215006, P.R. China
}

Received November 30, 2017; Accepted June 28, 2018

DOI: $10.3892 / \mathrm{mmr} .2018 .9386$

\begin{abstract}
MicroRNAs (miRNA/miRs) serve crucial roles in the progression of human glioblastoma (GBM); however, the exact regulatory mechanisms of miRNAs in human GBM remain unclear. The present study aimed to investigate the roles of miR-454-3p in human GBM. Reverse transcription-quantitative polymerase chain reaction (RT-qPCR) analysis was performed to examine the expression of miR-454-3p in glioma tissues and adjacent tissues. Human GBM cell lines (LN-229, A172 and GL15) and a normal human astrocyte cells (HA1800) were used for analysis. In addition, RT-qPCR and western blotting were applied for mRNA and protein expression analysis, respectively. The cell proliferation was measured using a Cell Counting kit- 8 assay. Furthermore, scratch and Transwell assays were employed for the analysis of cell migration and invasion. A luciferase reporter assay was used to verify the target of miR-454-3p. The results revealed that miR-454-3p was downregulated in the glioma tissues and GBM cell lines, including LN-229, A172 and GL15. Additionally, the overexpression of miR-454-3p significantly suppressed the proliferation, migration and invasion of LN-229 cells. Furthermore, cytoplasmic polyadenylation element-binding protein 1 (CPEB1) was confirmed as a direct target of miR-454-3p. These findings indicated that the overexpression of miR-454-3p inhibited cell proliferation, migration and invasion by downregulating CPEB1. Therefore, miR-454-3p may act as a tumor suppressor and represent an effective therapeutic strategy in GBM.
\end{abstract}

\section{Introduction}

Glioblastoma (GBM) is one of the most common types of malignant tumor and is a prevalent type of primary cancer of

Correspondence to: Dr Shiming Zhang, Department of Neurosurgery, The First Affiliated Hospital of Soochow University, 188 Shizi Street, Suzhou, Jiangsu 215006, P.R. China

E-mail: bright257@yeah.net

Key words: glioblastoma, microRNAs, cytoplasmic polyadenylation element-binding protein 1 , tumor suppressor the central nervous system in adults (1-4). Developments in the treatments for GBM, including chemotherapy with temozolomide, radiotherapy, and combination therapy with tumor resection have been made; in patients with newly diagnosed malignant glioma, improvements in the 2-year survival rate (from 11 to $27 \%$ ), the 3 -year survival rate (from 4 to $16 \%$ ) and the 5-year survival rate (from 2 to $10 \%$ ) were observed in $2014(5,6)$. However, these survival rates remain relatively low; the underlying molecular mechanisms of GBM-associated tumorigenesis, chemotherapy resistance, progression and metastasis require further investigation. Therefore, it is of clinical importance that novel therapeutic targets and biomarkers associated with GBM are identified.

MicroRNAs (miRNA/miRs) are a class of small non-coding RNAs of 19-24 nucleotides in length that serve important regulatory roles in post-transcriptional gene expression (7,8). Additionally, downregulation of miR-454-3p has been identified in certain types of cancer in humans, including pancreatic ductal adenocarcinoma (9) and breast cancer (10), while upregulation of miR-454 has been observed in other types of cancer, including hepatocellular carcinoma, non-small cell lung cancer (11) and colorectal cancer (12). In pancreatic ductal adenocarcinoma, it has been reported that miR-454 is able to regulate stromal cell proliferation, thereby controlling the growth of pancreatic ductal adenocarcinoma (13). Additionally, in osteosarcoma, miR-454 functions as a tumor suppressor gene that suppresses cell proliferation and invasion by directly targeting c-Met (14). In hepatocellular carcinoma, miR-454 functions as an oncogene by inhibiting chromodomain-helicase-DNA-binding protein 5, resulting in the inhibition of tumor cell proliferation and invasion (15). Additionally, in uveal melanoma, miR-454 is upregulated and promotes cell proliferation, colony formation, invasion and induction of the cell cycle (16). Most relevant to the present study, a recent investigation into GBM revealed that miR-454 was able to inhibit cell proliferation by suppressing pyruvate dehydrogenase kinase 1 expression (17); however, the role and underlying mechanisms of miR-454 downregulation in GBM are largely unknown.

Cytoplasmic polyadenylation element-binding protein 1 (CPEB1) is a sequence-specific RNA-binding protein that regulates mRNA translation by dynamically controlling the length of the poly(A) tail length of an mRNA transcript (18). The biological roles of CPEB1-mediated regulation include 
the control of cell cycle progression, cellular senescence and inflammation (19-21). Additionally, CPEB1 has been considered to serve as a tumor suppressor gene. For instance, a recent study reported that CPEB1 mediated epithelial-to-mesenchymal transition (EMT) and breast cancer metastasis by regulating the apical localization of tight junction protein $\mathrm{ZO}-1$ mRNA (22). In melanoma, miR-455-5p has been reported to promote melanoma metastasis via inhibition of CPEB1 (23); in glioma, knockdown of CPEB1 reduces cell senescence by regulating the expression and distribution of cellular tumor antigen p53, and CPEB1 was also demonstrated to be directly regulated by the tumor suppressor miR-101 (24).

The results of the present study revealed that the overexpression of miR-454 in GBM cells in vitro inhibited cell proliferation, migration and invasion. Importantly, the present study also observed that miR-454-3p negatively regulated the target gene CPEB1. Thus, the present study investigated whether miR-454-3p and CPEB1 may be a novel therapeutic target for the treatment of patients with GBM.

\section{Patients and methods}

Patient and tissue samples. A total of 30 human glioma and matched adjacent tissues were collected between January 2015 and December 2016 at Huai'an First People's Hospital (Huai'an, China). The median age of the patients was 51 years, and the age ranged from 29-73 years and there were 19 male and 11 female. All tissue samples were collected for analysis upon obtaining informed consent from all patients. In addition, patients did not receive chemotherapy, radiotherapy or any other treatments prior to surgery. The present study was approved by the Research Ethics Committee of Huai'an First People's Hospital (Huai'an, China).

Cell culture. Human GBM cell lines (LN-229, A172 and GL15) and normal human astrocyte cells (HA1800) were obtained from the Shanghai Institutes for Biological Sciences (Chinese Academy of Sciences, Shanghai, China). Adherent cultures of LN-229 and HA1800 cell lines were maintained in high-glucose Dulbecco's modified Eagle's medium (DMEM; Thermo Fisher Scientific, Inc., Waltham, MA, USA) supplemented with $10 \%$ fetal bovine serum (FBS; Beijing Solarbio Science and Technology Co., Ltd., Beijing, China). All cells were cultured in a humidified incubator at $37^{\circ} \mathrm{C}$ in an atmosphere of $5 \% \mathrm{CO}_{2}$.

Cell transfection. The human GBM cells (LN-229) were seeded in 6 -well plates $\left(2 \times 10^{5}\right.$ cells/well $) 1$ day prior to transfection, which was conducted when cells reached $60-70 \%$ confluence. The LN-229 cells were untransfected or transfected with mimic control or miR-454-3p mimic (Shanghai GenePharma Co., Ltd., Shanghai, China) at a concentration of $40 \mathrm{nM}$, and the untreated cells served as the control. The successful transfection was determined by RT-qPCR. The sequences of miR-454-3p was: 5'-UAGUGCAAUAUUGCUUAUAGG GU-3', miR-454-3p mimic was: 5'-UAGUGCAAUAUUGCU UAUAGGGU-3 and mimic control was: 5'-UCACAACCU CCUAGAAAGAGUAGA-3'. Then the cells were incubated for $24 \mathrm{~h}$ to continue the further analysis. Transfection with the miRNAs and with the plasmids for the luciferase assay (described below) was performed using Lipofectamine ${ }^{\circledR} 2000$ (Invitrogen; Thermo Fisher Scientific, Inc.), according to the manufacturer's protocol.

RNA extraction and reverse transcription-quantitative polymerase chain reaction ( $R T-q P C R)$. Human GBM cell lines (LN-229, A172 and GL15) and normal human astrocyte cells (HA1800) were lysed in TRIzol ${ }^{\circledR}$ reagent (Invitrogen; Thermo Fisher Scientific, Inc.) for total RNA extraction; a total of $1 \mu \mathrm{g}$ total RNA was used for cDNA synthesis with an RNA PCR kit (Takara Biotechnology Co., Ltd., Dalian, China). For cDNA synthesis, samples were incubated at $42^{\circ} \mathrm{C}$ for $30 \mathrm{~min}, 99^{\circ} \mathrm{C}$ for $5 \mathrm{~min}$ and $5^{\circ} \mathrm{C}$ for $5 \mathrm{~min}$. qPCR was conducted using an ABI7500 Real-Time PCR Instrument (Applied Biosystems; Thermo Fisher Scientific, Inc.). The qPCR conditions were as follows: Pre-denaturation at $95^{\circ} \mathrm{C}$ for $5 \mathrm{~min}$, followed by initiation at $94^{\circ} \mathrm{C}$ for $30 \mathrm{sec}$, annealing at $60^{\circ} \mathrm{C}$ for $30 \mathrm{sec}$, and elongation at $75^{\circ} \mathrm{C}$ for $1.5 \mathrm{~min}$ for 32 cycles, following which samples were stored at $4^{\circ} \mathrm{C}$. Hairpin-it ${ }^{\mathrm{TM}}$ of miR-454-3p and U6 RT-qPCR Primer Set (Shanghai GenePharma Co., Ltd.) were used to measure the relative quantity of miR-454-3p; the expression of miR-454 was normalized to the endogenous expression of U6. The primers used were as follows: miR-454-3p forward, 5'-CTC AACTGGTGTCGTGGAGTCGGCAATTCAGTTGAGACC CTATA-3' and reverse, 5'-ACACTCCAGCTGGGTAGTGCA ATATTGCTTA-3'; U6 forward, 5'-CTCGCTTCGGCAGCA CA-3' and reverse, 5'-AACGCTTCACGAATTTGCGT-3'. Additionally, metadherin, astrocyte elevated gene-1 (AEG-1) and matrix metalloproteinase-9 (MMP-9) RT-qPCR Primer Sets (Shanghai GenePharma Co., Ltd.) were used to measure the relative quantities of metadherin, AEG-1 and MMP-9, with normalization of the mRNA expression levels to the endogenous expression levels of GAPDH. The primers used were as follows: Metadherin forward, 5'-AAATGGGCGGAC TGTTGAAGT-3' and reverse, 5'-CTGTTTTGCACTGCT TTAGCAT-3'; AEG-1 forward, 5'-AAATGGGCGGACTGT TGAAGT-3' and reverse, 5'-CTGTTTTGCACTGCTTTA GCAT-3'; MMP-9 forward, 5'-GGGACGCAGACATCGTCA TC-3' and reverse, 5'-TCGTCATCGTCGAAATGGGC-3'; and GAPDH forward, 5'TGTGGGCATCAATGGATTTGG3' and reverse, 5'-ACACCATGTATTCCGGGTCAAT-3'. All reactions were performed in triplicate. The relative expression of genes was normalized to GAPDH. Data were analyzed using the $2^{-\Delta \Delta \mathrm{Cq}}$ method (25).

Cell Counting kit-8 (CCK-8) assay. Cellular proliferation was measured with a CCK-8 kit (Dojindo Molecular Technologies, Inc., Kumamoto, Japan), according to the manufacturer's protocol. LN-229 cells were assigned with untreated (control), mimic control and miR-454-3p mimic groups. At $24 \mathrm{~h}$ post-transfection, $100 \mu \mathrm{l}$ cells suspension were seeded into 96 -well plates at a density of $8 \times 10^{3}$ cells/well and incubated at $37^{\circ} \mathrm{C}$ for $6 \mathrm{~h}$. At the indicated time points (12, 24 and $48 \mathrm{~h}), 10 \mu \mathrm{l} \mathrm{CCK}-8$ solution was added to each well. Following incubation at $37^{\circ} \mathrm{C}$ for $1 \mathrm{~h}$, the absorbance was measured with a plate reader at $450 \mathrm{~nm}$.

Transwell and scratch assays of LN-229 cell migration and invasion. Transwell culture inserts (8-mm pore size; Falcon; BD Biosciences, Franklin Lakes, NJ, USA) were placed into 
the wells of 24-well culture plates, separating the upper and lower chambers. LN-229 cells were assigned with untreated (control), mimic control and miR-454-3p mimic groups. For the cell invasion assay, Matrigel (BD Biosciences) was used to pre-coat the upper side of the membrane, which was subsequently incubated at $37^{\circ} \mathrm{C}$ for $1 \mathrm{~h}$ for gel formation, and hydrated in FBS for $2 \mathrm{~h}$ prior to use. In the lower chamber, $600 \mu 1$ DMEM containing 10\% FBS was added, and the three groups cells were added to the upper chamber at a density of $1 \times 10^{5}$ cells/well respectively. Following incubation at $37^{\circ} \mathrm{C}$ for $24 \mathrm{~h}$. The level of migration was observed under an optical microscope (Leica DMI6000B; Leica Microsystems GmbH, Wetzlar, Germany), and counted for 5 random (magnification, x100) fields per well. Cell counts are expressed as the mean number of cells per field of view (26).

For the cell migration assay, the LN-229 cells in the logarithmic phase were cultured in DMEM in 24-well plates at a density of $10^{4}$ cells/well to obtain a monolayer culture. The monolayer was carefully scratched with a new 1-ml pipette tip across the center of the well, with the long axis of the tip remaining perpendicular to the bottom of the well, such that the width of the scratch was equal to the outer diameter of the tip. Following scratching, the cells were incubated at $37^{\circ} \mathrm{C}$ with $5 \% \mathrm{CO}_{2}$ for $24 \mathrm{~h}$. Images of the migrated cells were captured at 0 and $24 \mathrm{~h}$ and were captured from 5 random (magnification, $x 5$ ) fields in each sample.

Target prediction. According to TargetScan 7.1 (http://www. targetscan.org/vert_71/), it was predicted that CPEB1 may be targeted by miR-454-3p.

Luciferase reporter assays. For the luciferase reporter assay, LN-229 cells were seeded in 96-well plates at a density of $10^{4}$ cells/well and incubated at $37^{\circ} \mathrm{C}$ with $5 \% \mathrm{CO}_{2}$ for 24 h. Subsequently, pGL3-CPEB1-3'untranslated region (UTR)-wild type (WT) or pGL3-CPEB1-3'UTR-mutant plasmids (constructed by Beyotime Institute of Biotechnology, Haimen, China) were transfected into the control, miR-454-3p mimics and negative control miR-454-3p mimics groups using Lipofectamine 2000. The transfection efficiency was normalised with an internal control Renilla luciferase vector (pRL-CMV; Promega Corporation, Madison, WI, USA). Following $48 \mathrm{~h}$ incubation, a luciferase assay kit (cat. no. RG005; Beyotime Institute of Biotechnology) was used to measure the reporter activity according to the manufacturer's protocols.

Western blot analysis. After LN-229 cells were untransfected or transfected with mimic control or miR-454-3p mimic cells were washed with cold PBS and treated with a lysis buffer (cat. no. C3702; Beyotime Institute of Biotechnology). The quality was detected using BCA method and protein concentration was measured with a NanoDrop instrument (NanoDrop; Thermo Fisher Scientific, Inc., Pittsburgh, PA, USA). A total of $30 \mu \mathrm{g}$ protein samples were separated by $15 \%$ SDS-PAGE, and the proteins were transferred onto polyvinylidene difluoride membranes. Subsequently, the membranes were blocked with a buffer containing $10 \%$ non-fat milk in PBS with $0.05 \%$ Tween-20 for $2 \mathrm{~h}$ at room temperature, and then incubated with primary antibodies against metadherin,
AEG-1, MMP-9, CPEB1 and GAPDH for $1 \mathrm{~h}$ at room temperature. Additionally, the membranes were incubated at room temperature with horseradish peroxidase-labeled goat anti-rabbit antibody (1:1,000; cat. no. A0208; Beyotime Institute of Biotechnology, Haimen, China) conjugated to horseradish peroxidase for $45 \mathrm{~min}$. Protein bands were visualized by enhanced chemiluminescence (Abbott Laboratories, Arlington Heights, IL, USA). Evaluation of target protein expression was performed using ImageJ version 1.38 (National Institutes of Health, Bethesda, MD, USA).

The primary antibodies [anti-metadherin (1:500; cat. no. MA515564), MMP-9 (1:1,000; cat. no. PA516851), CPEB1 (1:1,000; cat. no. PA520561) and GAPDH (1:500; cat. no. PA519440)] were purchased from Wuhan Khayal Bio-Technology Co., Ltd. (Wuhan, China); anti-AEG-1 (1:1,000; cat. no. PL032231R) was purchased from Otwo Biotech (Shenzhen), Inc. (Shenzhen, China).

Statistical analysis. All results were confirmed in at least three independent experiments, and the qualitative data from single representative experiments are presented. All quantitative data are presented as the mean \pm standard deviation. Statistical analysis was performed using GraphPad Prism version 5.01 (GraphPad Software, Inc., La Jolla, CA, USA). A Student's t-test was used for comparisons between two groups. One-way analysis of variance followed by a least significant difference post hoc test was used for comparisons between multiple groups. $\mathrm{P}<0.05$ was considered to indicate a statistically significant difference.

\section{Results}

miR-454-3p expression is significantly downregulated in glioma tissues and GBM cell lines. To investigate the role of miR-454-3p in GBM development, the expression levels of miR-454-3p in tissue samples and GBM cell lines were analyzed by RT-qPCR. As presented in Fig. 1, it was observed that miR-454-3p levels were significantly lowered in glioma tissues and GBM cell lines (LN-229, A172 and GL15) compared with adjacent tissues and normal human astrocyte cells (HA1800) $(\mathrm{P}<0.01)$, respectively. From the results of Fig. 1, the miR-454-3p levels were significantly lower in LN-299 cell than in A172 and GL15 cells, so LN-229 cells were chosen for further analysis.

Overexpression of miR-454-3p inhibits the proliferation of $L N-229$ cells. To investigate whether miR-454-3p serves a role as a tumor suppressor in GBM, the effects of miR-454-3p overexpression on the proliferation of GBM cells were analyzed. LN-229 cells were transfected with control miR, miR-454-3p mimic or negative control miR-454-3p mimic, and the relative expression of $\mathrm{miR}-454-3 \mathrm{p}$ was verified by RT-qPCR (Fig. 2A). As was demonstrated in Fig. 2A, the miR-454-3p level was significantly increased following overexpression of miR-454-3p compared with the negative control miR-454-3p mimic $(\mathrm{P}<0.01)$. In addition, the effects of miR-454-3p on cell proliferation was investigated via a CCK-8 assay. Analysis of the results indicated that the overexpression of miR-454-3p significantly suppressed LN-229 cell proliferation compared with the negative control 
A

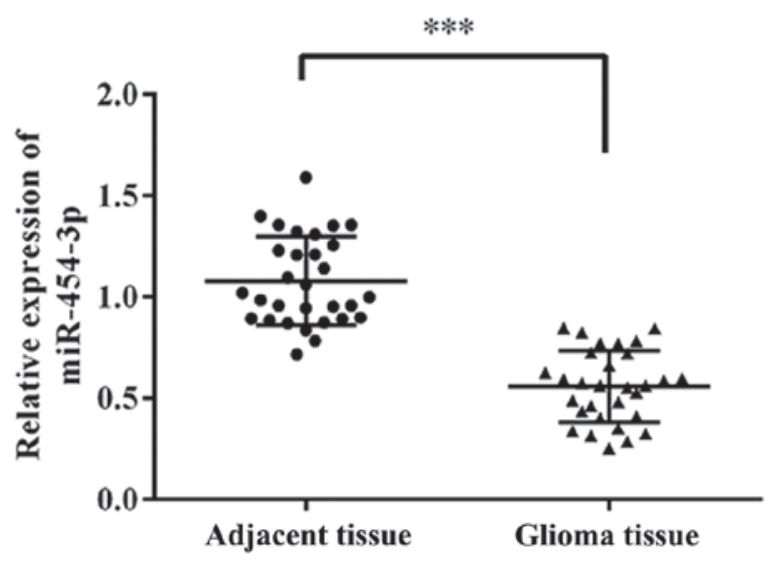

B

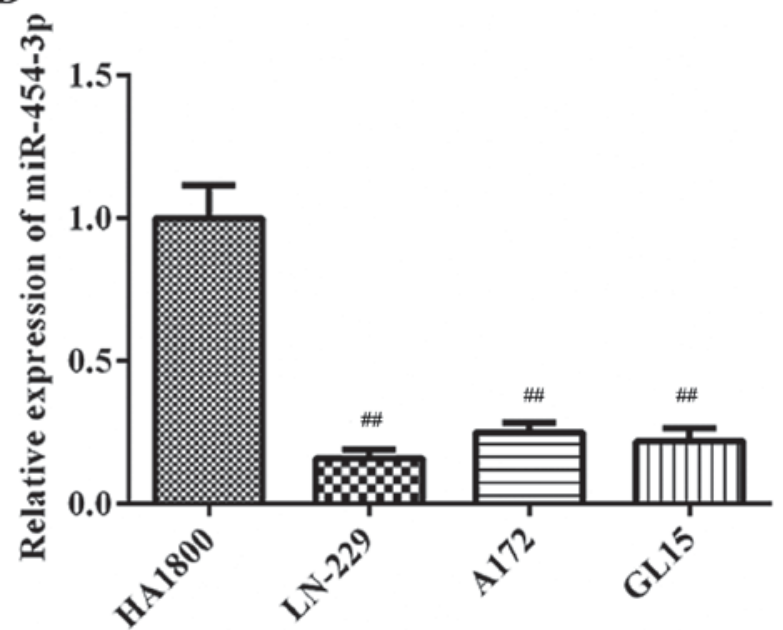

Figure 1. miR-454-3p expression is downregulated in glioma tissues and various human GBM cell lines. (A) Relative expression levels of miR-454-3p were determined by RT-qPCR in human glioma and adjacent tissues. (B) Relative expression levels of miR-454-3p were determined by RT-qPCR in human GBM cell lines (LN-229, A172 and GL15) and a normal human astrocyte cell line (HA1800). ${ }^{\# \#} \mathrm{P}<0.01$ vs. HA1800 and ${ }^{* * *} \mathrm{P}<0.001$. GBM, glioblastoma; miR, microRNA; RT-qPCR, reverse transcription-quantitative polymerase chain reaction.
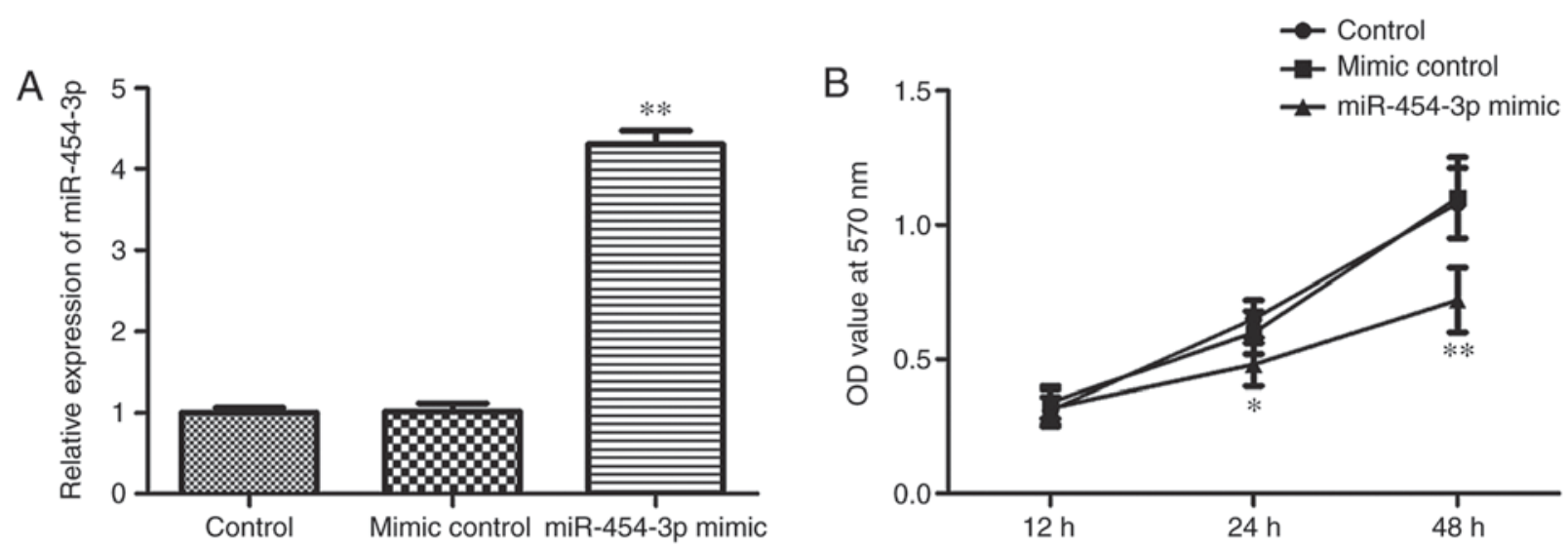

Figure 2. Overexpression of miR-454-3p inhibits the proliferation of LN-229 cells. LN-229 cells were divided into three transfection groups: Control, mimic control and miR-454-3p mimic groups. (A) Relative expression levels of miR-454-3p were measured by reverse transcription-quantitative polymerase chain reaction. (B) Cell proliferation was assessed by a Cell Counting kit- 8 assay; the OD value of each group in LN-229 cells was determined at $570 \mathrm{~nm}$. "P<0.05, ${ }^{* *} \mathrm{P}<0.01$ vs. mimic control. miR, microRNA; OD, optical density.

group (Fig. 2B). Above date suggested that miR-454-3p overexpression could inhibit the proliferation of LN-229 cells.

Overexpression of miR-454-3p inhibits the invasion of $L N-229$ cells. As migration and invasion are two key stages associated with malignant progression and metastasis (27), the effects of miR-454-3p on the migration and invasion of LN-229 cells was investigated in the present study. The results of the Transwell and scratch assays indicated that the overexpression of miR-454-3p significantly inhibited the invasion and migration of LN-229 cells compared with the negative control group (Figs. 3 and 4). Collectively, these results suggested that miR-454-3p overexpression exerted tumor suppressive effects by suppressing the proliferation, migration and invasion of the LN-229 GBM cell line.

Metadherin has been reported to regulate mesenchymal marker protein expression in numerous types of tumor and to promote cancer metastasis (28-30). Additionally, AEG-1 has been reported to serve a pivotal oncogenic role in tumorigenesis $(31,32)$, and MMPs, a family of zinc-binding proteins, including MMP-9, have been demonstrated to serve important roles in tumor cell invasion and metastasis due to their ability to degrade the extracellular matrix $(33,34)$. In the present study, western blotting and RT-qPCR revealed that miR-454-3p significantly decreased the protein and mRNA expression levels of CPEB1, metadherin, AEG-1 and MMP-9 compared with in the control group (Fig. 5).

CPEB1 is a target gene of miR-454-3p. The TargetScan database was used to identify the potential target mRNA of miR-454-3p in the present study. The results demonstrated that CPEB1 was a putative target gene of miR-454-3p (Fig. 6A). Subsequently, a dual-luciferase activity assay revealed that miR-454-3p significantly suppressed the luciferase activity of the WT 3'-UTR of CPEB1 compared with that of the mutant 
A
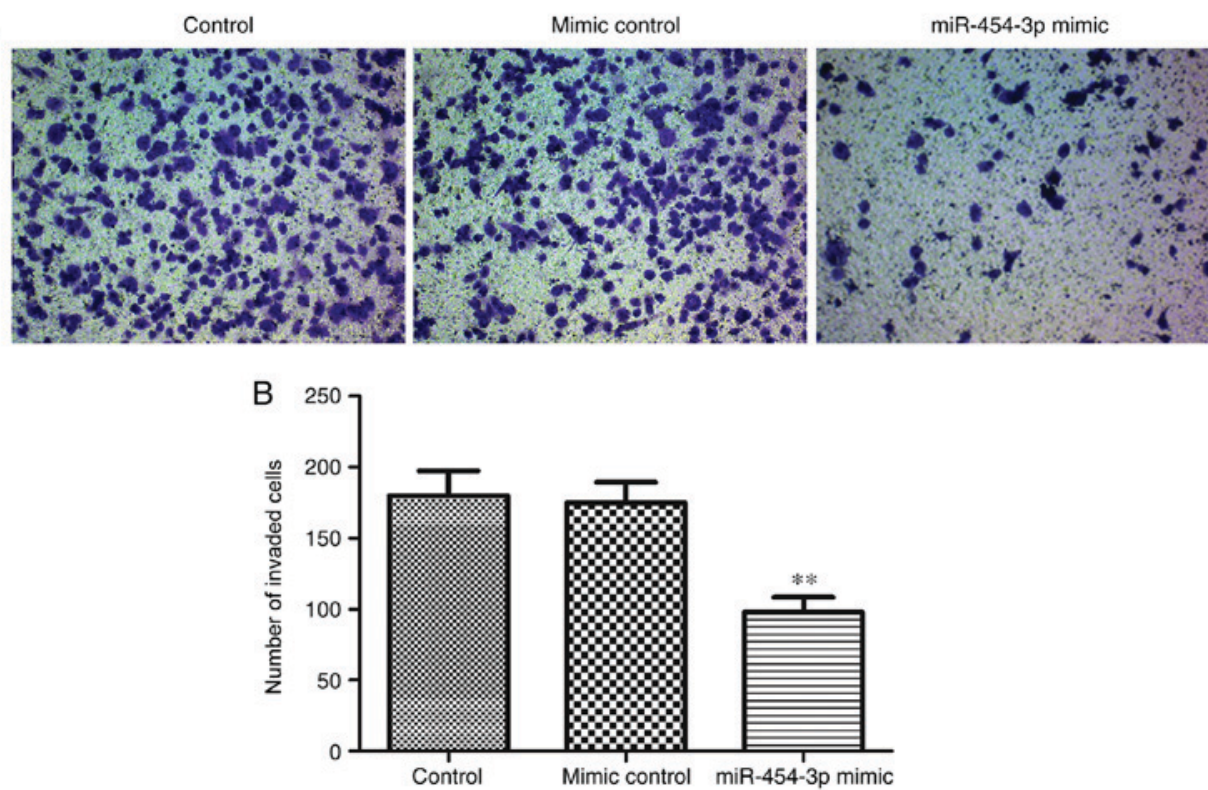

Figure 3. Overexpression of miR-454-3p inhibits the invasion of LN-229 cells. Cell invasion was assessed with a Transwell assay. (A) Images of cell invasion of LN-229 cells (magnification, $x 100$ ). (B) Number of invading LN-229 cells as determined with a counting chamber. ${ }^{* *} \mathrm{P}<0.01 \mathrm{vs}$. mimic control. miR, microRNA.

A
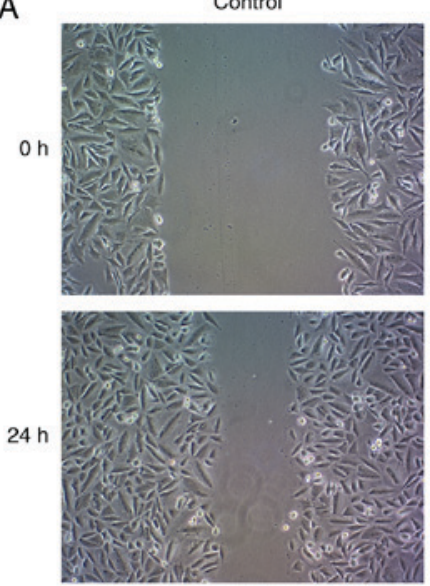

Mimic control
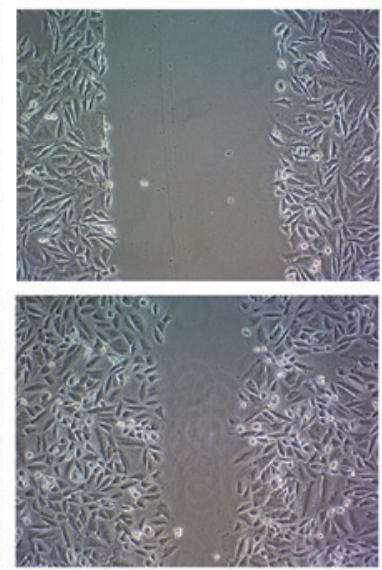

miR-454-3p mimic
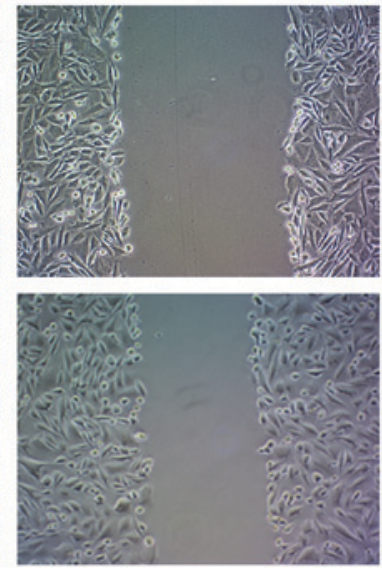

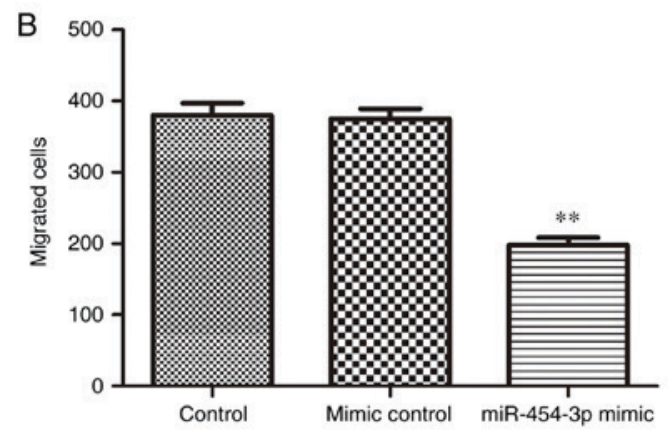

Figure 4. Overexpression of miR-454-3p inhibits the migration of LN-229 cells. The migratory activity of LN-229 cells was identified with a scratch assay. (A) Images of the migration of LN-229 cells at 0 and $24 \mathrm{~h}$ (magnification, $\mathrm{x} 4$ ). (B) Migrated distance of LN-229 cells after $24 \mathrm{~h}$ incubation. ${ }^{* * *} \mathrm{P}<0.01 \mathrm{vs}$. mimic control. miR, microRNA.

type (Fig. 6B). Furthermore, the overexpression of miR-454-3p was observed to suppress the protein expression of CPEB1 in the LN-229 cells by western blot analysis (Fig. 5B). These data indicated that CPEB1 is a direct target of miR-454-3p in GBM LN-229 cells.

\section{Discussion}

GBM is among the most lethal and aggressive forms of brain cancer, and accounts for $15 \%$ of brain malignancies (35). Despite technological improvements in the diagnosis and 

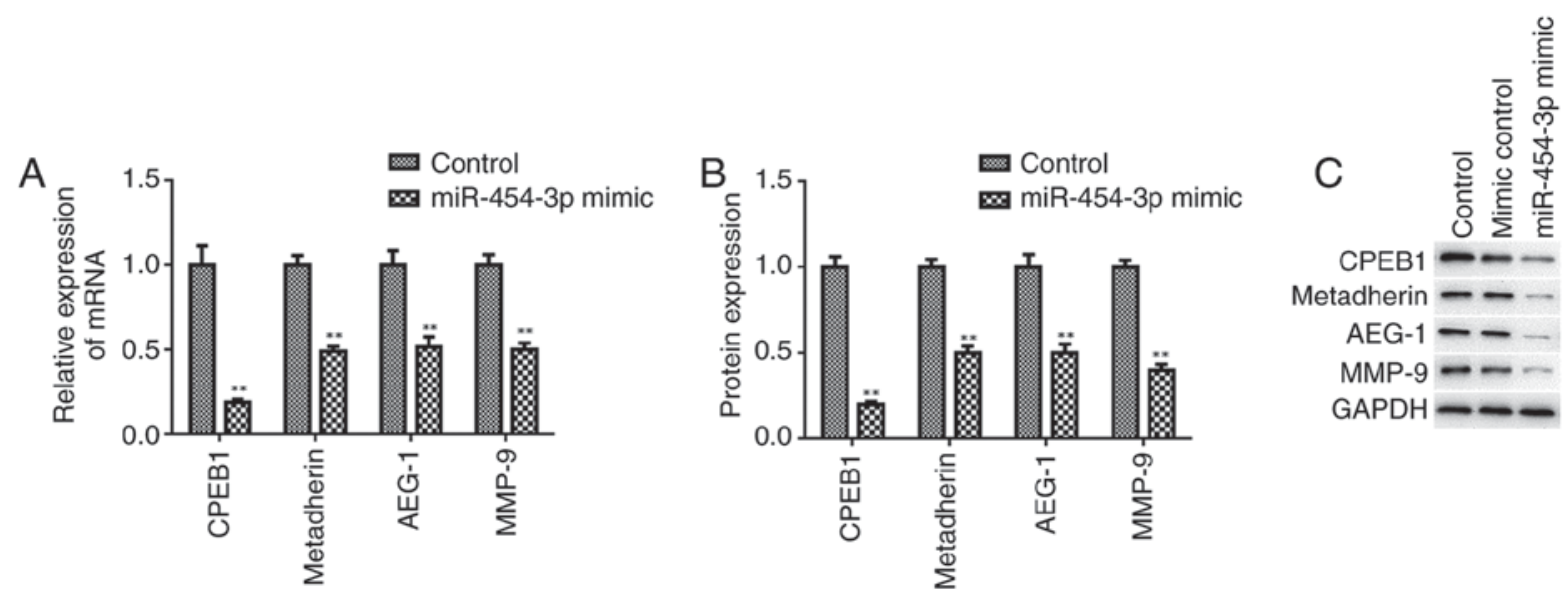

Figure 5. Overexpression of miR-454-3p inhibits the expression of tumor metastasis-associated proteins. The relative expression levels of mRNA were quantified by reverse transcription-quantitative polymerase chain reaction and protein expression levels were determined by western blotting. (A) Relative mRNA expression levels of CPEB1, metadherin, AEG-1 and MMP-9 in LN-229 cells. (B) Protein expression profiles of CPEB1, metadherin, AEG-1 and MMP-9 in LN-229 cells. (C) Western blotting image of the protein bands for CPEB1, metadherin, AEG-1, MMP-9 and internal reference GAPDH in LN-229 cells. ${ }^{* *} \mathrm{P}<0.01$ vs. respective control. AEG-1, astrocyte elevated gene-1; CPEB1, cytoplasmic polyadenylation element-binding protein 1; miR, microRNA; MMP-9, matrix metalloproteinase-9.

treatment of GBM, the rates of treatment failure and recurrence remain an issue. The poor prognosis of patients with GBM is primarily due to late diagnoses and poor responses to chemotherapy (36). Therefore, further investigation is urgently required to identify and develop novel therapeutic approaches for the prevention and treatment of GBM.

Recent studies have revealed that miRNAs contribute to the progression and development of GBM $(37,38)$. For example, decreased expression levels of miR-146a in GBM have been associated with the regulation of cell proliferation and apoptosis via the targeting of notch 1 (39). In addition, upregulated miR-622 has been observed to inhibit cell proliferation, motility and invasion by repressing KRAS proto-oncogene GTPase in GBM (40). Additionally, miR-10b in GBM may mediate TGF- $\beta 1$-regulated cell proliferation, migration and EMT (41); however, to the best of our knowledge, the roles of miR-454-3p, which is located on chromosome $17 \mathrm{q} 22$, in the progression of GBM, particularly its effects on cell proliferation and migration and target gene regulation, have not yet been studied.

In the present study, downregulation of miR-454-3p in glioma tissues and GBM cell lines was observed. Functional experiments further revealed that the overexpression of miR-454-3p may inhibit the proliferation, migration and invasion of the LN-229 GBM cell line. Recent studies have reported that miR-454 may act as an oncogene or tumor suppressor in cancer $(17,42)$. For instance, miR-454-3p was reported to be upregulated and to function as an oncogene in hepatocellular carcinoma, non-small cell lung cancer (11) and colorectal cancer (12); however, the present study revealed that miR-454-3p served a tumor suppressing role in GBM LN-229 cells. Thus, miR-454-3p may serve a variety of roles in different types of tumor. To the best of our knowledge, there have been no reports regarding the association between miR-454-3p and CPEB1. The results of the present study indicated that miR-454-3p negatively regulated CPEB1, and identified CPEB1 as a direct target of miR-454-3p. Therefore, the findings of the present study collectively indicated that miR-454-3p may be an important miRNA that is potentially

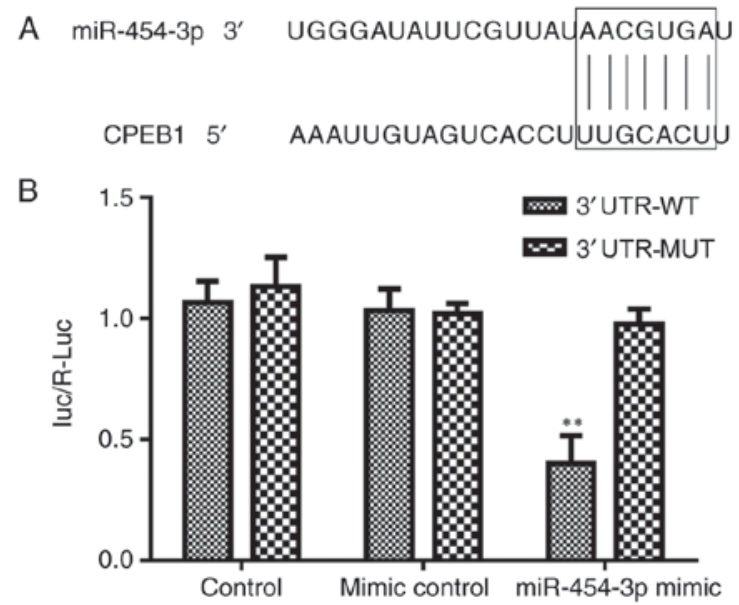

Figure 6. CPEB1 is a target gene of miR-454-3p. (A) Predicted miR-454-3p target sequence in the 3 '-UTR of CPEB1. (B) Luciferase activity in control, mimic control and miR-454-3p group. ${ }^{* *} \mathrm{P}<0.01$ vs. 3' UTR-MUT group. CPEB1, cytoplasmic polyadenylation element-binding protein 1; R-luc, Renilla luciferase; miR, microRNA; Mut, mutant; UTR, untranslated region; WT, wild type.

associated with the proliferation, migration and invasion of GBM cells.

Metastasis is one of the principal factors that contributes to the mortality of patients with GBM (43). The present study reported that the overexpression of miR-454-3p inhibited the migration and invasion of GBM cells in vitro. In epithelial cancers, EMT is regarded as one of the major mechanisms that promote cell migration, invasion and metastasis. A recent study demonstrated that CPEB1 mediates EMT and metastasis in breast cancer (22). In the present study, miR-454-3p was suggested to inhibit the migration and invasion of GBM cells by negatively regulating CPEB1. In addition, MMPs are proteolytic enzymes that serve a pivotal role in the transformation and progression of tumors at all stages, particularly invasion and metastasis (44). The present study revealed that within GBM cells, miR-454-3p significantly inhibited the 
expression of MMP-9. Collectively, these results indicated that the upregulation of miR-454-3p in GBM cells suppressed the migration and invasion of the tumor cells by inhibiting CPEB1 and MMP-9 expression.

In conclusion, the present study demonstrated that miR-454-3p was significantly downregulated in GBM cell lines, and that miR-454-3p overexpression suppressed cell proliferation, migration and invasion, potentially by targeting CPEB1 in the GBM cells. These findings indicated a novel tumor suppressive role of miR-454 in the development of GBM. Furthermore, miR-454-3p and CPEB1 may be potential therapeutic targets in the treatment of GBM.

\section{Acknowledgements}

The authors of the present study would like to thank the Chinese Academy of Sciences (Shanghai, China) for providing the cell lines and Shanghai GenePharma Co., Ltd. (Shanghai, China) for their support with cell transfection.

\section{Funding}

No funding was received.

\section{Availability of data and materials}

The analyzed data sets generated during the present study are available from the corresponding author on reasonable request.

\section{Authors' contributions}

$\mathrm{XH}$ and SZ searched the literature and designed the study. $\mathrm{XH}$ and $\mathrm{YW}$ performed the experiments and interpreted the data. YW contributed to the materials. XH wrote and revised the manuscript. All authors read and approved the final manuscript.

\section{Ethics approval and consent to participate}

The present study was approved by the Research Ethics Committee of Huai'an First People's Hospital. All tissue samples were collected for used after obtaining informed consent from all patients.

\section{Patient consent for publication}

Not applicable.

\section{Competing interests}

The authors declare that they have no competing interests.

\section{References}

1. Siegel RL, Miller KD and Jemal A: Cancer statistics,2017. CA Cancer J Clin 67: 7-30, 2017.

2. Furnari FB, Fenton T, Bachoo RM, Mukasa A, Stommel JM, Stegh A, Hahn WC, Ligon KL, Louis DN, Brennan C, et al: Malignant astrocytic glioma: Genetics, biology, and paths to treatment. Genes Dev 21: 2683-2710, 2007.

3. Meyer MA: Malignant gliomas in adults. N Engl J Med 359: 1850,2008
4. Fisher JL, Schwartzbaum JA, Wrensch M and Wiemels JL: Epidemiology of brain tumors. Neurol Clin 25: 867-890, 2007.

5. Stupp R, Hegi ME, Mason WP, van den Bent MJ, Taphoorn MJ, Janzer RC, Ludwin SK, Allgeier A, Fisher B, Belanger K, et al: Effects of radiotherapy with concomitant and adjuvant temozolomide versus radiotherapy alone on survival in glioblastoma in a randomised phase III study: 5-year analysis of the EORTC-NCIC trial. Lancet Oncol 10: 459-466, 2009.

6. Yao C, Lv S, Han M, Zhang J, Zhang Y, Zhang L, Yi R, Zhuang D and $\mathrm{Wu} \mathrm{J}$ : The association of Crk-like adapter protein with poor prognosis in glioma patients. Tumor Biol 35: 5695-5700, 2014.

7. Bartel DP: MicroRNAs: Genomics, biogenesis, mechanism, and function. Cell 116: 281-297, 2004.

8. Irwandi RA and Vacharaksa A: The role of microRNA in periodontal tissue: A review of the literature. Arch Oral Biol 72: 66-74, 2016.

9. Fan Y, Shi C, Li T and Kuang T: microRNA-454 shows anti-angiogenic and anti-metastatic activity in pancreatic ductal adenocarcinoma by targeting LRP6. Am J Cancer Res 7: 139-147, 2017.

10. Cao ZG, Li JJ, Yao L, Huang YN, Liu YR, Hu X, Song CG and Shao ZM: High expression of microRNA-454 is associated with poor prognosis in triple-negative breast cancer. Oncotarget 7: 64900-64909, 2016.

11. Zhu DY, Li XN, Qi Y, Liu DL, Yang Y, Zhao J, Zhang CY, Wu K and Zhao S: MiR-454 promotes the progression of human non-small cell lung cancer and directly targets PTEN. Biomed Pharmacother 81: 79-85, 2016.

12. Liang HL, Hu AP, Li SL, Xie JP, Ma QZ and Liu JY: miR-454 prompts cell proliferation of human colorectal cancer cells by repressing CYLD expression. Asian Pac J Cancer Prev 16: 2397-2402, 2015

13. Fan Y, Xu LL, Shi CY, Wei W, Wang DS and Cai DF: MicroRNA-454 regulates stromal cell derived factor-1 in the control of the growth of pancreatic ductal adenocarcinoma. Sci Rep 6: 22793, 2016.

14. Niu G, Li B, Sun J and Sun L: miR-454 is down-regulated in osteosarcomas and suppresses cell proliferation and invasion by directly targeting c-Met. Cell Prolif 48: 348-355, 2015.

15. Yu L, Gong X, Sun L, Yao H, Lu B and Zhu L: miR-454 functions as an oncogene by inhibiting CHD5 in hepatocellular carcinoma. Oncotarget 6: 39225-39234, 2015.

16. Sun L, Wang Q, Gao X, Shi D, Mi S and Han Q: MicroRNA-454 functions as an oncogene by regulating PTEN in uveal melanoma. FEBS Lett 589: 2791-2796, 2015.

17. Fang B, Zhu J, Wang Y, Geng F and Li G: MiR-454 inhibited cell proliferation of human glioblastoma cells by suppressing PDK1 expression. Biomed Pharmacother 75: 148-152, 2015.

18. Richter JD: CPEB: A life in translation. Trends Biochem Sci 32: 279-285, 2007.

19. Groisman I, Jung MY, Sarkissian M, Cao Q and Richter JD: Translational control of the embryonic cell cycle. Cell 109: 473-483, 2002.

20. Ivshina M, Lasko P and Richter JD: Cytoplasmic polyadenylation element binding proteins in development, health, and disease. Annu Rev Cell Dev Biol 30: 393-415, 2014.

21. Udagawa T, Farny NG, Jakovcevski M, Kaphzan H, Alarcon JM, Anilkumar S, Ivshina M, Hurt JA, Nagaoka K, Nalavadi VC, et al: Genetic and acute CPEB1 depletion ameliorate fragile X pathophysiology. Nat Med 19: 1473-1477, 2013.

22. Nagaoka K, Fujii K, Zhang H, Usuda K, Watanabe G, Ivshina M and Richter JD: CPEB1 mediates epithelial-to-mesenchyme transition and breast cancer metastasis. Oncogene 35: 2893-2901, 2016.

23. Shoshan E, Mobley AK, Braeuer RR, Kamiya T, Huang L, Vasquez ME, Salameh A, Lee HJ, Kim SJ, Ivan C, et al: Reduced adenosine-to-inosine miR-455-5p editing promotes melanoma growth and metastasis. Nat Cell Biol 17: 311-321, 2015.

24. Xiaoping L, Zhibin Y, Wenjuan L, Zeyou W, Gang X, Zhaohui L, Ying Z, Minghua W and Guiyuan L: CPEB1, a histone-modified hypomethylated gene, is regulated by miR-101 and involved in cell senescence in glioma. Cell Death Dis 4: e675, 2013.

25. Livak KJ and Schmittgen TD: Analysis of relative gene expression data using real-time quantitative PCR and the 2(-Delta Delta C((T) method. Methods 25: 402-408, 2001.

26. Zhang L, Dong Y, Zhu N, Tsoi H, Zhao Z, Wu CW, Wang K, Zheng S, Ng SS, Chan FK, et al: microRNA-139-5p exerts tumor suppressor function by targeting NOTCH1 in colorectal cancer. Mol Cancer 13: 124, 2014. 
27. Sttg PS: New insights into the tumor metastatic process revealed by gene expression profiling. Am J Pathol 166: 1291-1294, 2005.

28. Du Y, Jiang B, Song S, Pei G, Ni X, Wu J, Wang S, Wang Z and Yu J: Metadherin regulates actin cytoskeletal remodeling and enhances human gastric cancer metastasis via epithelial-mesenchymal transition. Int J Oncol 51: 63-74, 2017.

29. Tong L, Chu M, Yan B, Zhao W, Liu S, Wei W, Lou H, Zhang S, $\mathrm{Ma}$ S, Xu J and Wei L: MTDH promotes glioma invasion through regulating miR-130b-ceRNAs. Oncotarget 8: 17738-17749, 2017.

30. Hou Y, Yu L, Mi Y, Zhang J, Wang K and Hu L: Association of MTDH immunohistochemical expression with metastasis and prognosis in female reproduction malignancies: A systematic review and meta-analysis. Sci Rep 6: 38365, 2016.

31. Wang J, Chen X, and Tong M: Knockdown of astrocyte elevated gene-1 inhibited cell growth and induced apoptosis and suppressed invasion in ovarian cancer cells. Gene 616: 8-15, 2017.

32. Liu X, Lv Z, Zou J, Liu X, Ma J, Sun C, Sa N and Xu W: Elevated AEG-1 expression in macrophages promotes hypopharyngeal cancer invasion through the STAT3-MMP-9 signaling pathway. Oncotarget 7: 77244-77256, 2016.

33. Skerenova M, Mikulova V, Capoun O, Zima T and Tesarova P: Circulating tumor cells and serum levels of MMP-2, MMP-9 and VEGF as markers of the metastatic process in patients with high risk of metastatic progression. Biomed Pap Med Fac Univ Palacky Olomouc Czech Repub 161: 272-280, 2017.

34. Shin SS, Song JH, Hwang B, Noh DH, Park SL, Kim WT, Park SS, Kim WJ and Moon SK: HSPA6 augments garlic extract-induced inhibition of proliferation, migration, and invasion of bladder cancer EJ cells; Implication for cell cycle dysregulation, signaling pathway alteration, and transcription factor-associated MMP-9 regulation. PLoS One 12: e0171860, 2017.

35. Young RM, Jamshidi A, Davis G and Sherman JH: Current trends in the surgical management and treatment of adult glioblastoma. Ann Transl Med 3: 121, 2015.
36. Krishnatry R, Zhukova N, Guerreiro Stucklin AS, Pole JD, Mistry M, Fried I, Ramaswamy V, Bartels U, Huang A, Laperriere $\mathrm{N}$, et al: Clinical and treatment factors determining long-term outcomes for adult survivors of childhood low-grade glioma: A population-based study. Cancer 122: 1261-1269, 2016.

37. Zhang X, Zhang X, Hu S, Zheng M, Zhang J, Zhao J, Zhang X, Yan B, Jia L, Zhao J, et al: Identification of miRNA-7 by genome-wide analysis as a critical sensitizer for TRAIL-induced apoptosis in glioblastoma cells. Nucleic Acids Res 45: 5930-5944, 2017.

38. Xiuju C, Zhen W and Yanchao S: SOX7 inhibits tumor progression of glioblastoma and is regulated by miRNA-24. Open Med (Wars) 11: 133-137, 2016.

39. Hu HQ, Sun LG and Guo WJ: Decreased miRNA-146a in glioblastoma multiforme and regulation of cell proliferation and apoptosis by target notch1. Int J Biol Markers 31: e270-275, 2016.

40. Wang $X, X i n ~ Z, X u ~ Y$ and Ma J: Upregulated miRNA-622 inhibited cell proliferation, motility, and invasion via repressing kirsten rat sarcoma in glioblastoma. Tumour Biol 37: 5963-5970, 2016.

41. Ma C, Wei F, Xia H, Liu H, Dong X, Zhang Y, Luo Q, Liu Y and Li Y: MicroRNA-10b mediates TGF- $\beta 1$-regulated glioblastoma proliferation, migration and epithelial-mesenchymal transition. Int J Oncol 50: 1739-1748, 2017.

42. Zhou, L, Qu YM, Zhao XM and Yue ZD: Involvement of miR-454 overexpression in the poor prognosis of hepatocellular carcinoma. Eur Rev Med Pharmacol Sci 20: 825-829, 2016.

43. Fidler IJ: The pathogenesis of cancer metastasis the: Seed and soil' hypothesis revisited. Nat Rev Cancer 3: 453-458, 2003.

44. Tabouret E, Boudouresque F, Farina P, Barrié M, Bequet C, Sanson M and Chinot O: MMP2 and MMP9 as candidate biomarkers to monitor bevacizumab therapy in highgrade glioma. Neuro Oncol 17: 1174-1176, 2015. 